\title{
Analyse numérique du régime THD dans un patin échelon
}

\author{
Comparaison entre les modèles de Reynolds \\ et de Navier-Stokes
}

\author{
Mihai B. Dobrica - Michel Fillon \\ Laboratoire de Mécanique des Solides - UMR CNRS 6610 \\ Université de Poitiers \\ Boulevard Marie et Pierre Curie, Téléport 2, BP 30179 \\ F-86962 Futuroscope Chasseneuil cedex \\ \{dobrica,fillon\}@lms.univ-poitiers.fr
}

\begin{abstract}
RÉSUMÉ. La littérature sur la lubrification hydrodynamique manque de modèles thermohydrodynamiques (THD) adaptés aux géométries discontinues. La méthode la plus souvent employée, celle des différences finies, est difficilement applicable à la simulation des domaines d'écoulement non continus. Le présent article propose deux modèles basés sur la méthode des volumes finis et applicables aux domaines discontinus. Le premier algorithme, ayant à la base l'équation de Reynolds généralisée, manque de précision au voisinage de la discontinuité, mais il nous offre, en revanche, une bonne vitesse de calcul. Le deuxième modèle, basé sur les équations de Navier-Stokes, plus précis mais aussi plus lent, est ensuite proposé. Une comparaison des deux modèles appliqués dans le cas simple d'un patin échelon, en 2D, sera enfin effectuée. Différentes configurations géométriques seront analysées. Notre étude finit par la validation du modèle de Reynolds pour la plupart des configurations rencontrées en lubrification fluide.

ABSTRACT. A lack of thermohydrodynamic (THD) models adapted to discontinuous fluid film geometries can be noted in the lubrication-related literature. The finite differences method, largely used in THD analysis, cannot be easily applied to discontinuous flow domains. This article proposes two finite volumes based models, suitable for discontinuous domains. The first model, based on Reynolds generalized equation, lacks in accuracy near the discontinuity line, but gains in computing speed. The second model, based on Navier-Stokes equations, is more accurate but also significantly more demanding in terms of computing requirements. The two models are applied and compared on the simple geometry of a bi-dimensional Rayleigh step bearing. Different configurations are analyzed, allowing the validation of the Reynolds model for most of the cases encountered in fluid film lubrication.

MOTS-CLÉS: lubrification hydrodynamique, patin échelon, effets thermiques, volumes finis. KEYWORDS: hydrodynamic lubrication, Rayleigh step bearing, thermal effects, finite volumes method.
\end{abstract}

DOI:10.3166/REMN.16.683-701 @ 2007 Lavoisier, Paris

REMN - 16/2007. Modélisations numériques en mécanique, pages 683 à 701 


\section{Introduction}

L'analyse thermohydrodynamique (THD) des butées et des paliers ne constitue plus une nouveauté aujourd'hui. Après l'introduction de l'équation de Reynolds généralisée par Dowson, (1962), des études complètes ont été menées sur la plupart des configurations géométriques rencontrées. Ezzat et al. (1973) ont proposé l'une des premières solutions THD numériques pour un blochet de longueur finie, avec des conditions limites réalistes, à l'aide de la méthode des différences finies. Boncompain et al. (1986) ont amélioré le modèle numérique, prenant en compte l'écoulement inverse, la rupture du film fluide et les déformations dues à l'échauffement du solide, dans le cas d'un palier lisse. L'algorithme, toujours en différences finies, prend en compte la direction d'écoulement mais reste toutefois instable si la zone de recirculation devient importante. Une solution similaire est utilisée par Fillon et al., (1996) dans la construction des cartes de température des paliers à patins oscillants. L'une des premières études THD en volumes finis est présentée par Han et al., (1990). Ils ont résolu l'équation d'énergie complète pour un palier lisse de longueur finie, en obtenant une bonne convergence, même si l'écoulement inverse devient important. Hatakenaka et al. (2002) proposent une autre approche THD en volumes finis, basée cette fois-ci sur l'équation simplifiée de l'énergie, appliquée à un palier à deux lobes. Ils montrent aussi que l'approche en volumes finis ne pose plus de problèmes de convergence.

En ce qui concerne les géométries présentant des discontinuités dans l'épaisseur du film lubrifiant, les études sont peu nombreuses. Une étude THD basée sur les équations de Navier-Stokes couplées à l'équation de l'énergie est menée par Keogh et al., (1997), dans le cas d'un palier à rainures axiales. L'algorithme SIMPLEC est utilisé pour résoudre les équations de Navier-Stokes, avec une discrétisation en volumes finis. Toutefois, la discontinuité engendrée par les rainures n'est pas considérée dans le modèle numérique. Une solution similaire, présentant un traitement du phénomène de cavité mais négligeant aussi les discontinuités est proposée par Gandjalikhan et al., (2002). Récemment, He et al., (2004) ont réalisé une étude THD d'un palier à saut. La discontinuité est prise en compte lors de la résolution de l'équation de Reynolds généralisée, et une forme simplifiée de l'équation de l'énergie a été utilisée. Celle-ci néglige la variation de la température selon l'épaisseur du film, et les échanges thermiques avec les surfaces solides.

Cependant, les discontinuités dans l'épaisseur du fluide ont été mieux prises en compte dans quelques études hydrodynamiques (HD), en régime isotherme. Parmi celles-là, Braun et al., $(1993$; 1994) ont mené plusieurs études sur l'écoulement dans une poche hydrostatique située en-dessous d'une paroi mobile, à l'aide des techniques « Computational Fluid Dynamics » (CFD). La résolution des équations de Navier-Stokes leur a permis de bien décrire l'écoulement particulier engendré par les discontinuités. Arghir et al. (2002), lors d'une étude HD d'un patin échelon, introduisent des facteurs de perte de pression dans l'équation de Reynolds classique. Ces facteurs permettent de compenser les effets de l'inertie sur la ligne de 
discontinuité, tout en préservant les hypothèses de film mince. Cependant, la détermination de ces facteurs (selon la géométrie ou les paramètres de fonctionnement) reste un exercice difficile, qui requiert une comparaison avec une solution exacte (Navier-Stokes).

La plupart des études THD menées jusqu'à présent sont soit appliquées aux géométries continues soit elles négligent les discontinuités. La seule exception, (He et al., 2004), utilise un modèle thermique non réaliste car les variations de température selon l'épaisseur du film sont négligées, bien que leur importance ait été souvent montrée (Boncompain et al., 1986 ; Fillon et al., 1996 ; Han et al., 1990 ; Hatakenaka et al., 2002 ; Keogh et al., 1997). Cette absence d'études sur les géométries discontinues peut être expliquée par les difficultés de modélisation d'une telle géométrie avec la méthode des différences finies (très souvent utilisée). En plus, l'habilité d'un modèle basé sur l'équation de Reynolds généralisée d'analyser des domaines discontinus reste à montrer, au moins en régime thermohydrodynamique.

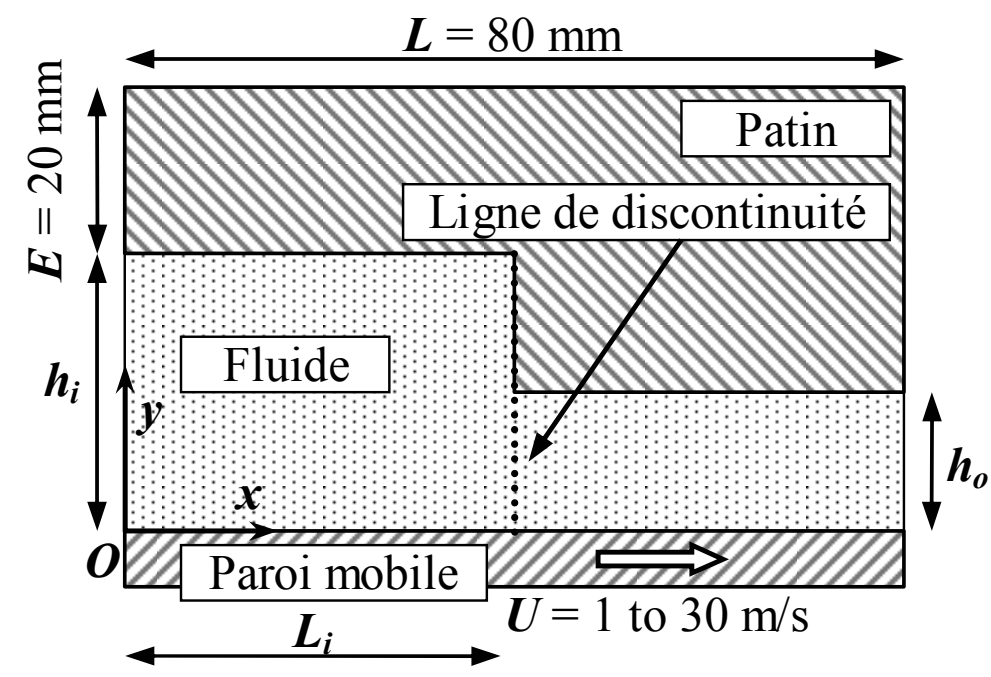

Figure 1. Géométrie du patin échelon

Cet article vient compléter les études existantes, avec une comparaison entre les modèles numériques de Reynolds et de Navier-Stokes, appliqués sur la géométrie simple d'un patin échelon, en 2D. Lors de cette comparaison, la solution obtenue avec le modèle basé sur les équations de Navier-Stokes est considérée exacte, et l'erreur introduite en utilisant le modèle simplifié de Reynolds est évaluée. Les paramètres géométriques et de fonctionnement seront variables, permettant ainsi d'établir les limites de validité du modèle de Reynolds. 


\section{Modèle théorique}

La forme étudiée est celle d'un patin échelon (patin de Rayleigh) de largeur supposée infinie (2D), présentée sur la figure 1. Les équations nécessaires pour l'analyse THD d'un tel problème peuvent être séparées en deux groupes : modélisation des paramètres hydrodynamiques et modélisation des aspects thermiques. Pour l'analyse hydrodynamique, deux modèles sont utilisés dans cette étude. Le premier modèle, plus simple, fait appel à l'équation de Reynolds généralisée [1] pour déterminer la distribution de la pression (Boncompain et al., 1986).

$$
\frac{\partial}{\partial x}\left(G \frac{\partial p}{\partial x}\right)=U \frac{\partial}{\partial x}\left(h-\frac{I_{2}}{J_{2}}\right)
$$

Ensuite, à partir de ce champ de pression, le champ de vitesses est déduit d'une manière similaire à celle présentée par (Boncompain et al., 1986) (équations [2], [3]). L'équation [2] permet de déterminer la composante longitudinale $u$ de la vitesse, la composante verticale $v$ étant déduite après, à l'aide de l'équation de continuité [3].

$$
\begin{aligned}
& u=\frac{\partial p}{\partial x}\left(I_{1}-\frac{I_{2} J_{1}}{J_{2}}\right)+U\left(1-\frac{J_{1}}{J_{2}}\right) \\
& \frac{\partial u}{\partial x}+\frac{\partial v}{\partial y}=0
\end{aligned}
$$

Dans les équations [1] et [2], la variation de la viscosité avec la température du fluide est prise en compte dans les intégrales transversales $G, I_{1,2}, J_{1,2}$, définies par la suite :

$$
\begin{array}{ll}
G=\int_{0}^{h} \frac{y}{\mu}\left(y-\frac{I_{2}}{J_{2}}\right) d y \\
I_{1}=\int_{0}^{y} \frac{\eta}{\mu} d \eta & I_{2}=\int_{0}^{h} \frac{y}{\mu} d y \\
J_{1}=\int_{0}^{y} \frac{1}{\mu} d \eta & J_{2}=\int_{0}^{h} \frac{1}{\mu} d y
\end{array}
$$


Dû à la réduction brusque de l'épaisseur du film introduite par l'échelon, une perte de pression se produit sur la ligne de discontinuité. Cette perte de pression, expliquée par les effets d'inertie, n'est pas prise en compte dans le modèle de Reynolds généralisé (les effets d'inertie sont complètement négligés dans ce modèle). Une méthode d'évaluation de ces pertes, toujours dans le cadre d'une analyse simplifiée avec l'équation de Reynolds, a été proposée dans la référence (Arghir et al., 2002). Ainsi, une equation additionnelle - l'équation de Bernoulli généralisée [4] - est appliquée sur la ligne de discontinuité. La perte de pression peut alors être calculée en fonction des vitesses moyennes du fluide juste avant et après la discontinuité :

$$
p^{-}+\frac{\rho\left(u^{-}\right)^{2}}{2}=p^{+}+\frac{\rho\left(u^{+}\right)^{2}}{2}+\xi \frac{\rho\left[M A X\left(u^{-}, u^{+}\right)\right]^{2}}{2}
$$

Le deuxième modèle utilisé dans l'analyse hydrodynamique est basé sur les équations de Navier-Stokes [5], [6] et sur l'équation de continuité [3]. La résolution couplée de ces équations permet d'obtenir des champs de pression et de vitesses plus précis, l'inertie étant prise en compte ; ceci est obtenu aux dépens d'un temps de calcul plus long.

$$
\begin{aligned}
& \left\{\begin{array}{l}
\rho\left(\frac{\partial u u}{\partial x}+\frac{\partial v u}{\partial y}\right)=\frac{\partial}{\partial x}\left(\mu \frac{\partial u}{\partial x}\right)+\frac{\partial}{\partial y}\left(\mu \frac{\partial u}{\partial y}\right)-\frac{\partial p}{\partial x}+s_{U} \\
\rho\left(\frac{\partial u v}{\partial x}+\frac{\partial v v}{\partial y}\right)=\frac{\partial}{\partial x}\left(\mu \frac{\partial v}{\partial x}\right)+\frac{\partial}{\partial y}\left(\mu \frac{\partial v}{\partial y}\right)-\frac{\partial p}{\partial y}+s_{V}
\end{array}\right. \\
& \left\{\begin{array}{l}
s_{U}=\frac{\partial}{\partial x}\left(\mu \frac{\partial u}{\partial x}\right)+\frac{\partial}{\partial y}\left(\mu \frac{\partial v}{\partial x}\right) \\
s_{V}=\frac{\partial}{\partial x}\left(\mu \frac{\partial u}{\partial y}\right)+\frac{\partial}{\partial y}\left(\mu \frac{\partial v}{\partial y}\right)
\end{array}\right.
\end{aligned}
$$

L'analyse thermique implique les mêmes équations, quel que soit le modèle hydrodynamique choisi. Ainsi, l'équation de l'énergie [7] est utilisée afin de déterminer la distribution de la température dans le fluide. L'équation de Laplace [8], une forme particulière de l'équation de l'énergie dont les termes convectifs et les termes source sont nuls, est appliquée dans le patin. Sa résolution permet l'évaluation de la température du patin, ainsi que l'évaluation de la quantité de chaleur évacuée par convection vers l'air ambiant. 


$$
\begin{aligned}
& \rho C_{p}\left(u \frac{\partial T}{\partial x}+v \frac{\partial T}{\partial y}\right)=K_{f}\left(\frac{\partial^{2} T}{\partial y^{2}}+\frac{\partial^{2} T}{\partial x^{2}}\right)+\mu\left(\frac{\partial u}{\partial y}\right)^{2} \\
& \frac{\partial^{2} T}{\partial x^{2}}+\frac{\partial^{2} T}{\partial y^{2}}=0
\end{aligned}
$$

La variation de la viscosité en fonction de la température du fluide peut être déterminée par la relation suivante, pour de faibles variations de température :

$$
\mu=\mu_{0} e^{-\beta\left(T-T_{0}\right)}
$$

Pour les deux modèles, les conditions aux limites du domaine fluide sont :

- pression nulle à l'entrée et à la sortie ;

- vitesse verticale nulle à l'entrée et à la sortie ;

- adhérence parfaite entre le fluide et les parois.

Du point de vue thermique, les conditions aux limites sont :

- température imposée $\left(T_{0}\right)$ dans la zone d'entrée ;

- continuité des flux thermiques à l'interface fluide/patin :

$$
\left.K_{f} \frac{\partial T}{\partial y}\right|_{y=h(x)^{-}}=-\left.K_{s} \frac{\partial T}{\partial y}\right|_{y=h(x)^{+}}
$$

- convection libre entre le patin et l'air ambiant :

$$
\begin{gathered}
\left.K_{s} \frac{\partial T}{\partial x}\right|_{x=0^{+}}=\left.h_{a}\left(T-T_{a}\right) \quad K_{s} \frac{\partial T}{\partial x}\right|_{x=L^{-}}=-h_{a}\left(T-T_{a}\right) \\
\left.K_{s} \frac{\partial T}{\partial y}\right|_{y=E^{-}}=-h_{a}\left(T-T_{a}\right)
\end{gathered}
$$

- bilan des flux thermiques nul à l'interface fluide/paroi mobile :

$$
\left.\int_{0}^{L} \frac{\partial T}{\partial y}\right|_{y=0^{+}} d x=0
$$




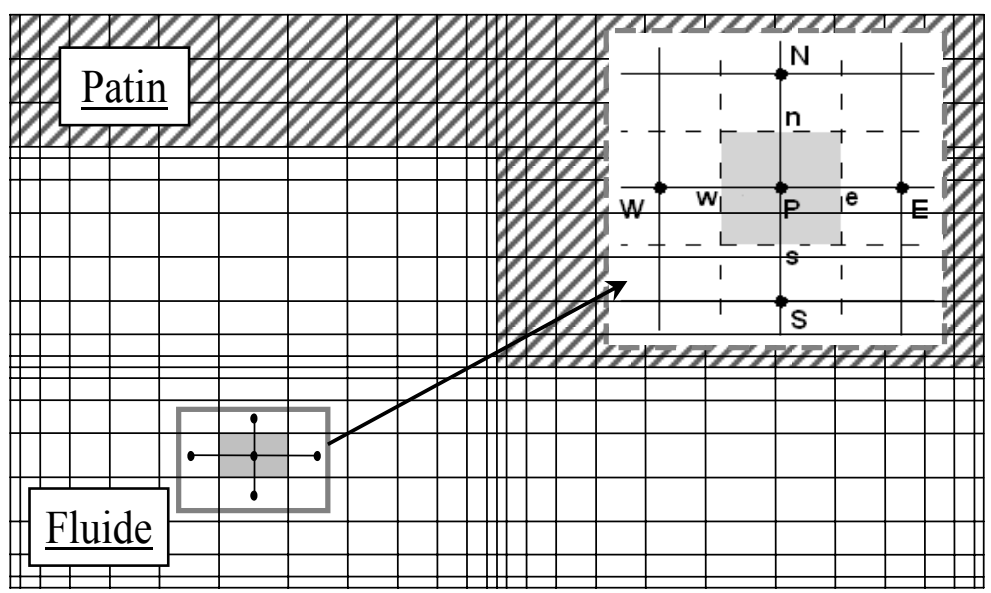

Figure 2. Maillage variable

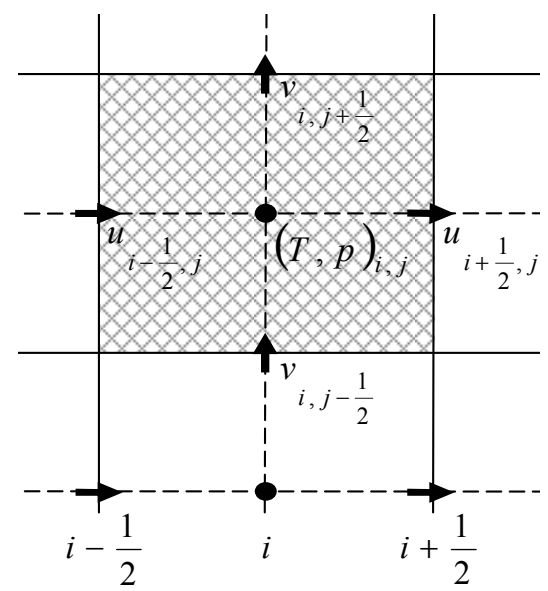

Figure 3. Maillage à distribution décalée des variables

\section{Modèle numérique}

Le domaine entier (fluide + patin) sera discrétisé avec un maillage cartésien variable selon les deux directions (figure 2). Dans la zone de la discontinuité, les éléments doivent être le plus carré possible, car les gradients de vitesse y sont importants. De même, avoir un maillage très fin selon x sur la ligne de discontinuité, où l'utilisation de l'équation de Reynolds génère des erreurs, permet de réduire l'aire affectée. De grands gradients de température existent au voisinage des parois, ce qui justifie le maillage variable selon $y$. Dans le patin, où seul le phénomène de diffusion 
thermique est présent, un maillage grossier peut être utilisé sans nuire à la précision du calcul. On parle alors de deux zones de maillage. La première, ayant $120 \times 60$ nœuds, inclut la totalité du domaine fluide plus une petite partie du patin (l'échelon). Cette zone a une épaisseur de quelques dizaines de micromètres, variable selon le cas étudié (l'épaisseur de cette zone est égale à l'épaisseur du film à l'entrée du patin). Une deuxième zone de maillage, ayant 120 x 30 éléments, couvre le patin solide (sauf l'échelon) et présente une épaisseur de 20 millimètres (pour les cas analysés par la suite). La taille du maillage à été choisie afin d'obtenir une précision suffisante (les erreurs estimées sont inférieures à $1 \%$ ) sans toutefois nuire à la vitesse de calcul.

Une distribution décalée des variables sera utilisée (Patankar, 1983), les nœuds de calcul des vitesses étant placés sur les côtés des éléments de pression et de température (figure 3). Cette méthode offre une meilleure évaluation des gradients des vitesses au centre des éléments (calcul de pression, température), et, en même temps, une précision améliorée dans le calcul des vitesses mêmes (dérivation de la pression sur un seul $\Delta x$ ). Un autre avantage d'une telle distribution de variables réside dans la facilité apportée lors de l'imposition des conditions limites. Utiliser des nœuds situés sur les frontières du domaine fluide (et pas à l'intérieur) pour les composantes de la vitesse permet d'imposer explicitement les vitesses des parois en ces points.
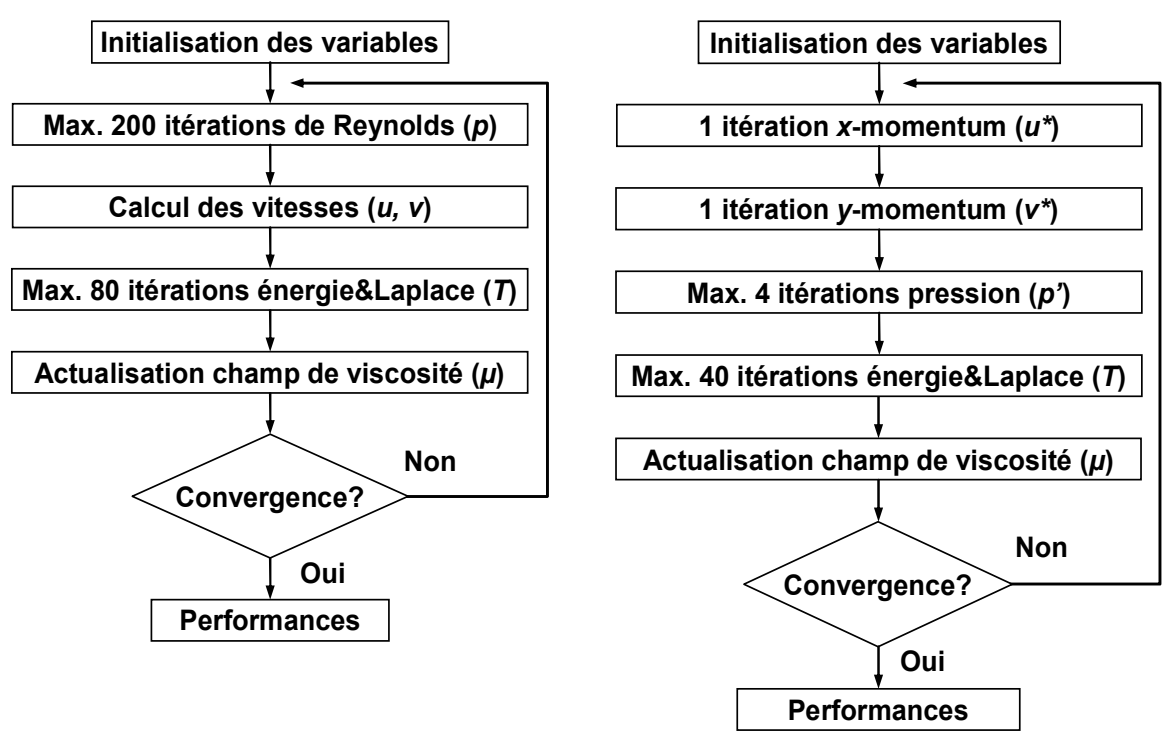

Figure 4. Organigrammes (Reynolds à gauche, Navier-Stokes à droite) 
La méthode des volumes finis a été choisie pour la résolution numérique des équations [1], [5-8]. De plus, le deuxième modèle requiert une solution couplée des équations de Navier-Stokes et de continuité, l'algorithme SIMPLE (Patankar, 1983) étant employé. Pour les équations de Navier-Stokes et de l'énergie (équations comportant aussi les phénomènes de conduction et convection à la fois) écrites avec des variables discrètes, le schéma "first order upwind» a été choisi. L'utilisation d'autres schémas de premier ordre (hybride, loi de puissance) n'a pas apporté une meilleure précision aux résultats. Les deux modèles nécessitent une solution couplée, similaire, qui alterne les itérations de calcul hydrodynamique et les itérations de calcul thermique, jusqu'à ce que la convergence soit obtenue pour toutes les équations. Le nombre des itérations effectuées pour chaque équation lors d'un pas global de calcul peut ainsi influencer la vitesse de convergence des deux modèles. Ces nombres sont donnés à titre indicatif dans les organigrammes présentés sur la figure 4, mais ils peuvent être variables d'un cas à l'autre afin d'améliorer la vitesse de convergence.

La résolution numérique est effectuée avec des codes de calcul écrits en Fortran 90. Deux méthodes itératives ont été utilisées pour la résolution des systèmes obtenus : la méthode Gauss-Seidel avec sur-relaxation (SOR) est utilisée pour les équations de Reynolds et de l'énergie, et la méthode SIP (Stone, 1968) est utilisée pour l'algorithme SIMPLE. Cette deuxième méthode présente l'avantage de ne pas réduire la vitesse de convergence lorsque les éléments de maillage sont trop allongés. Celle-ci est particulièrement adaptée à l'analyse numérique des films minces. Par ailleurs, elle est plus compliquée et aussi plus exigeante en termes de mémoire, le temps de calcul par itération étant plus élevé.

Les critères de convergence pour la partie hydrodynamique sont :

- variation inférieure à $10^{-8}$ dans la pression moyenne normalisée, entre deux itérations, pour l'algorithme de Reynolds généralisé ;

- termes source inférieurs a $10^{-3}$ pour l'algorithme de Navier-Stokes.

Pour la partie thermique, une variation inférieure à $10^{-8}$ de la température maximale entre deux itérations est requise. Afin que la convergence globale soit acceptée, il faut que les deux parties de l'algorithme (hydrodynamique et thermique) remplissent les critères de convergence en même temps. Les critères de convergence, présentés ci-après, peuvent être considérés conservateurs (plus d'itérations n'améliore pas la qualité de la solution).

La vitesse de convergence des deux algorithmes est fortement influencée par l'épaisseur du film lubrifiant. Lorsque celle-ci est faible, les effets thermiques sont plus prononcés, ce qui conduit à une diminution de la vitesse de convergence pour la partie thermique des algorithmes. Ainsi, le modèle de Reynolds généralisé converge en 2 minutes pour un film d'épaisseur de $500 \mu \mathrm{m}$ à la sortie, alors que 15 minutes de calculs sont nécessaires lorsque l'épaisseur n'est que de $10 \mu \mathrm{m}$. Les temps de convergence pour l'algorithme basé sur les équations de Navier-Stokes sont de 3 et 25 minutes, respectivement, sur un processeur ayant $3 \mathrm{GHz}$. 


\section{Résultats}

Les deux modèles numériques, dénotés par la suite Re (Reynolds) et NS (NavierStokes), ont été appliqués aux différentes configurations d'un patin échelon. Les caractéristiques géométriques et les paramètres de fonctionnement maintenus constants lors de l'étude numérique sont présentés dans le tableau 1. Pour les configurations analysées dans cette étude, le régime d'écoulement est laminaire ; les nombres de Reynolds maximaux sont inférieurs à 900 .

\begin{tabular}{|c|c|c|}
\hline \multicolumn{3}{|c|}{ Dimensions } \\
\hline Longueur & $L$ & $0,08 \mathrm{~m}$ \\
\hline Epaisseur du patin & $E$ & $0,02 \mathrm{~m}$ \\
\hline \multicolumn{3}{|c|}{ Propriétés du lubrifiant (ISO VG 32) } \\
\hline Viscosité à $40^{\circ} \mathrm{C}$ & $\mu_{40}$ & $0,03 \mathrm{~Pa} . \mathrm{s}$ \\
\hline Viscosité à $100^{\circ} \mathrm{C}$ & $\mu_{100}$ & $0,0045 \mathrm{~Pa} . \mathrm{s}$ \\
\hline Masse volumique & $\rho$ & $860 \mathrm{~kg} / \mathrm{m}^{3}$ \\
\hline Chaleur spécifique & $C_{\mathrm{p}}$ & $2000 \mathrm{~J} / \mathrm{kg} . \mathrm{K}$ \\
\hline Conduction thermique & $K_{\mathrm{f}}$ & $0,13 \mathrm{~W} /(\mathrm{m} . \mathrm{K})$ \\
\hline Température à l'entrée & $T_{0}$ & $40{ }^{\circ} \mathrm{C}$ \\
\hline Caractéristiques thermiques du patin et de l'air \\
\hline Conduction thermique & $K_{\mathrm{s}}$ & $45 \mathrm{~W} /(\mathrm{m} . \mathrm{K})$ \\
\hline Convection avec l'air & $h_{\mathrm{a}}$ & $20 \mathrm{~W} /\left(\mathrm{m}^{2} . \mathrm{K}\right)$ \\
\hline Température de l'air & $T_{\mathrm{a}}$ & $40^{\circ} \mathrm{C}$ \\
\hline
\end{tabular}

Tableau 1. Géométrie du patin

Dans un premier temps, une comparaison détaillée entre les champs de vitesses obtenus avec les deux modèles est présentée sur la figure 5, pour un patin fonctionnant avec un film d'épaisseur minimale $h_{\mathrm{o}}=20 \mu \mathrm{m}$, un rapport $h_{\mathrm{i}} / h_{\mathrm{o}}$ égal à 2 , un rapport $L_{\mathrm{i}} / L$ égal à 0.66 et une vitesse de la paroi mobile $U$ de $30 \mathrm{~m} / \mathrm{s}$. Cette comparaison permet de mettre en évidence les limites du modèle de Reynolds, lorsque celui-ci est appliqué à une configuration présentant des discontinuités dans l'épaisseur du film. Sur la ligne de discontinuité, le champ des vitesses obtenues avec le modèle de Reynolds présente des erreurs visibles (accélération ou décélération instantanée du fluide sur la ligne de discontinuité).

Ces erreurs sont mieux mises en évidence sur un maillage variable fin (200 éléments en direction longitudinale), permettant ainsi l'agrandissement d'une zone ayant $120 \mu \mathrm{m}$ en longueur, centrée sur la discontinuité. Cet agrandissement montre, d'une part, que les erreurs introduites par le modèle de Reynolds seront présentes même sur un maillage très fin, et d'autre part, que ces erreurs sont très 
localisées (en effet, à part une zone étroite centrée sur la discontinuité, les deux modèles conduisent au même champ de vitesses).

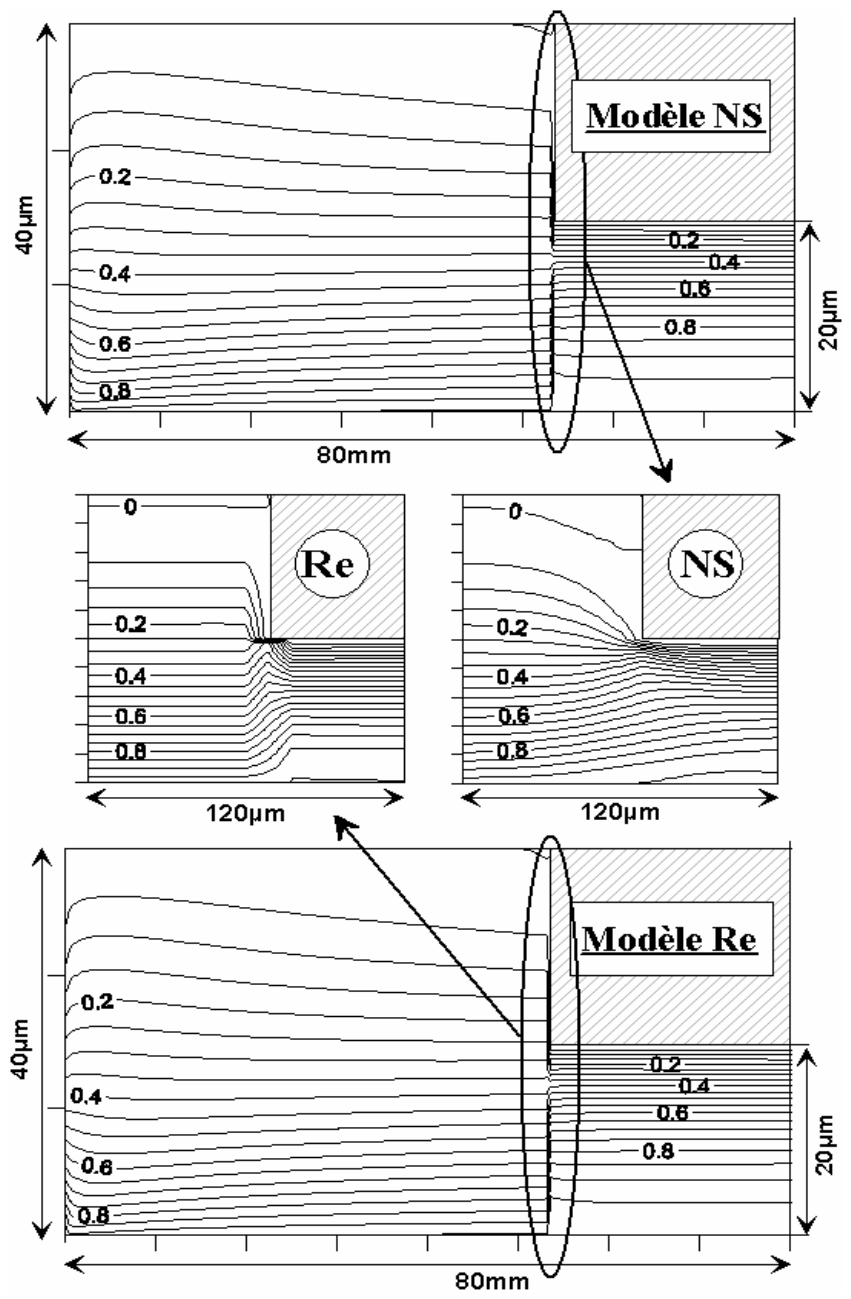

Figure 5. Lignes d'iso-vitesses u/U obtenues avec les deux modèles (Re et NS)

Pour la même configuration, la distribution de température obtenue avec le modèle NS est présentée sur la figure 6. On note l'échelle variable utilisée dans cette figure (mille fois réduite dans la zone supérieure du patin), afin d'obtenir une représentation détaillée du champ thermique à la fois dans le patin et dans le film. Cette échelle variable explique les discontinuités apparentes dans les isothermes, dans la zone de séparation entre le maillage fin (utilisé dans le fluide et dans la partie échelon du patin) et le maillage grossier (utilisé pour la majeure partie du patin). Le 
champ thermique calculé avec le modèle $\mathbf{R e}$ est très similaire à celui présenté, même dans la zone de discontinuité, prouvant ainsi que les erreurs localisées, observées sur les iso-vitesses, n'influencent pas la solution thermique.

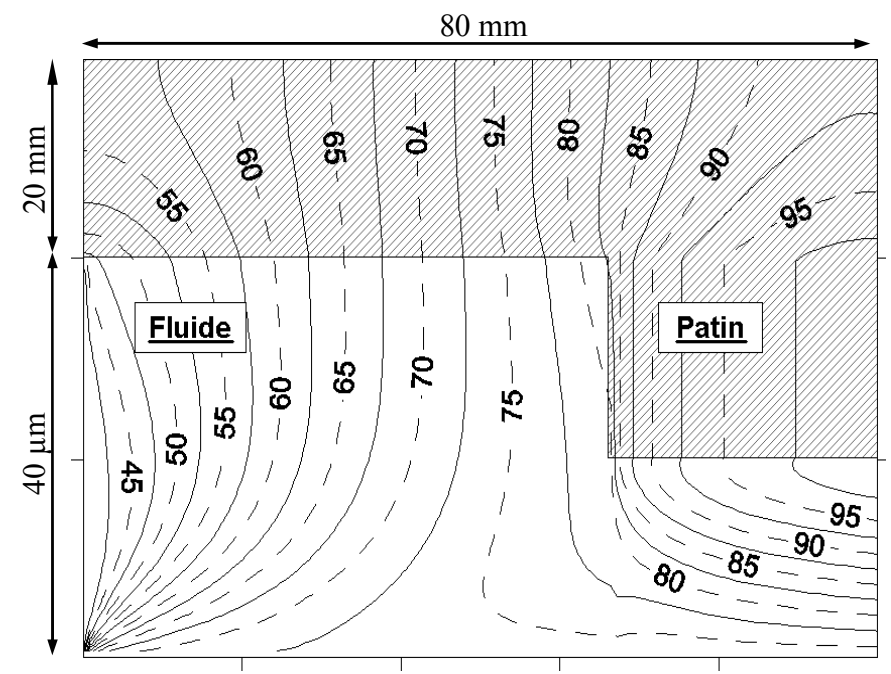

Figure 6. Distribution de température - modèle $\mathrm{NS}\left({ }^{\circ} \mathrm{C}\right)$

Par la suite, une étude comparative des deux modèles est effectuée pour différentes configurations d'un patin échelon, afin d'évaluer la précision du modèle de Reynolds lorsqu'il est appliqué à des domaines discontinus. Les valeurs des paramètres variables, telles l'épaisseur du film ou la vitesse de la paroi mobile, seront présentées sur les figures. Ces paramètres sont :

- le rapport entre la longueur du plan à l'entrée et la longueur du patin $L_{\mathrm{i}} / L$;

- le rapport des épaisseurs $h_{\mathrm{i}} / h_{\mathrm{o}}$;

- l'épaisseur minimale du film $h_{\mathrm{o}}$;

- la vitesse de la paroi mobile $U$.

La comparaison des deux modèles est effectuée au niveau des deux résultats du calcul :

- la température maximale atteinte dans le fluide, très significative du point de vue thermique ;

- la capacité de charge, caractérisant le champ de pression.

Sur les graphiques qui suivent, les écarts relatifs [9] entre la solution donnée par le modèle de Reynolds et celle obtenue avec le modèle de Navier-Stokes seront aussi présentés. Pour la plupart des cas analysés, le nombre de Reynolds moyen 
$R e=\rho U h_{\mathrm{i}} / \mu\left(T_{\text {moy }}\right)$, calculé dans la section d'entrée, sera mentionné afin de permettre la généralisation des résultats.

$$
\varepsilon_{T}=\frac{T^{\mathrm{Re}}-T^{\mathrm{NS}}}{T^{\mathrm{NS}}-T^{0}} \quad \varepsilon_{W}=\frac{W^{\mathrm{Re}}-W^{\mathrm{NS}}}{W^{\mathrm{NS}}}
$$

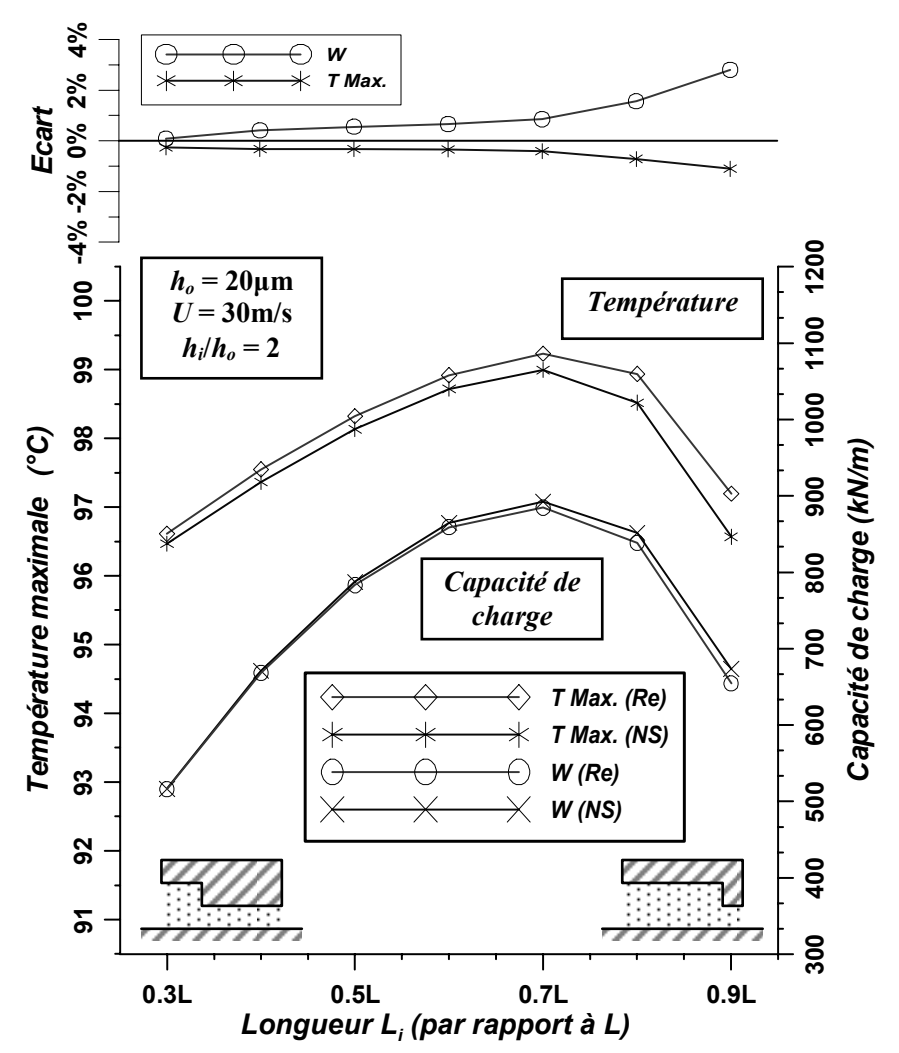

Figure 7. Influence du rapport des longueurs $L_{\mathrm{i}} / L$

Dans une première analyse (figure 7), le rapport des longueurs $L_{\mathrm{i}} / L$ varie de 0,3 à 0,9 . Dans ces cas, le nombre de Reynolds varie peu, sa valeur restant inférieure à $R e=100$. Les écarts entre les résultats des deux modèles, présentés au dessus du graphique, sont faibles, inférieurs à $3 \%$. Les écarts maximaux sont atteints pour un patin ayant une longueur du plan à l'entrée très importante, supérieure à 0,8 de la longueur totale du patin. La capacité de charge se montre optimale dans le cas $L_{\mathrm{i}} / L=0,66$. Nous avons donc retenu cette valeur pour les comparaisons effectuées par la suite. 
En variant le rapport entre l'épaisseur à l'entrée et celle à la sortie (figure 8), la corrélation des résultats reste bonne, avec un écart maximal de 1,5\%. La capacité de charge maximale est obtenue pour un rapport des épaisseurs $h_{\mathrm{i}} / h_{\mathrm{o}}$ égal à 3,5 (dans ce cas, la température maximale atteint $107^{\circ} \mathrm{C}$ et le nombre de Reynolds est égal à $R e=174)$. Apparemment, l'augmentation de ce rapport au-delà de 4 conduira à une baisse de la capacité de charge due à un échauffement plus prononcée du fluide. Dans ces deux premières analyses, l'épaisseur minimale du film à la sortie $(20 \mu \mathrm{m})$ et la vitesse de la paroi mobile $(30 \mathrm{~m} / \mathrm{s})$ ont été gardés constantes.

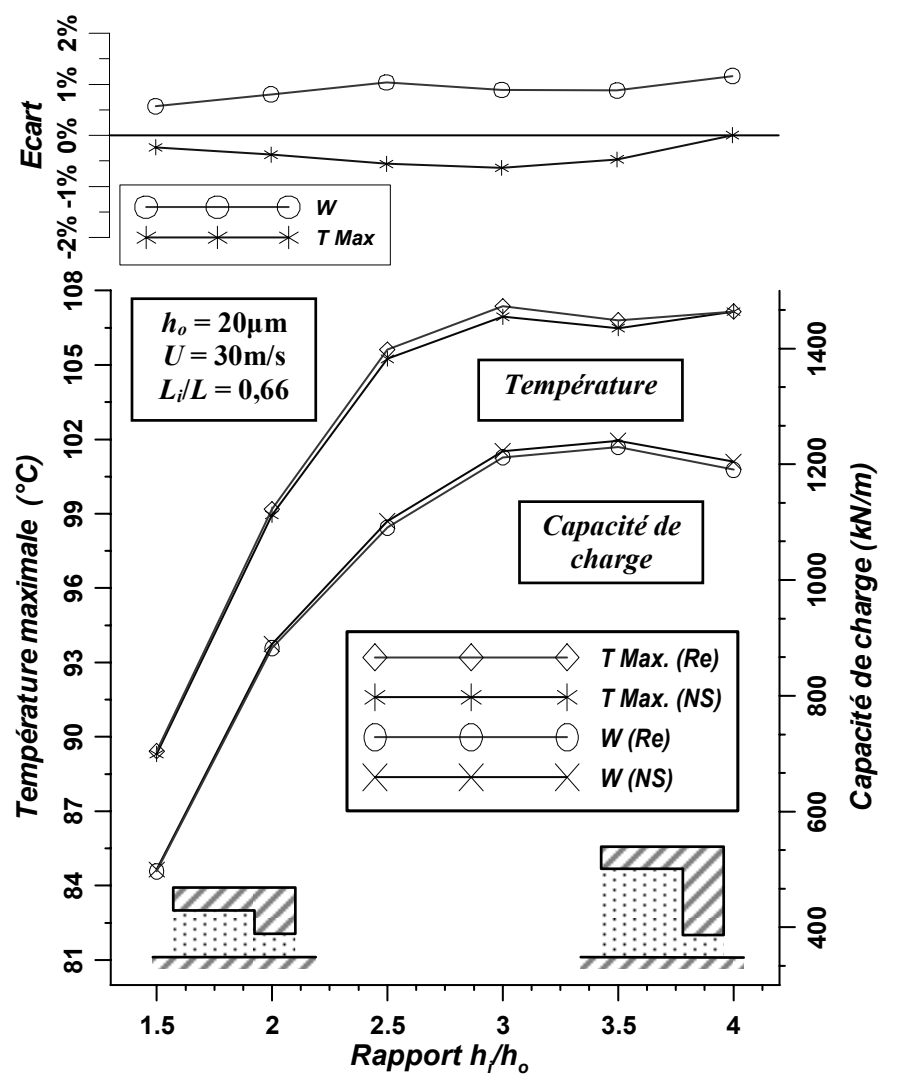

Figure 8. Influence du rapport des épaisseurs $h_{\mathrm{i}} / h_{\mathrm{o}}$

En variant l'épaisseur minimale du film tout en maintenant le rapport $h_{\mathrm{i}} / h_{\mathrm{o}}$ constant, des premiers écarts importants entre les deux modèles apparaissent (figure 9): pour une épaisseur du film supérieure à $120 \mu \mathrm{m}(R e=260)$, l'erreur générée par le modèle de Reynolds dépasse $5 \%$. Cette fois-ci, les erreurs sur la température maximale (relatives à la température d'alimentation $T_{0}$ ) sont plus importantes, avec un maximum de $25 \%$ pour une épaisseur minimale de $500 \mu \mathrm{m}$ 
( $R e=880)$. A cause du faible niveau d'échauffement lié à la forte épaisseur du film lubrifiant, ces écarts sont moins visibles sur les graphiques de température. Les écarts sur la capacité de charge ne dépassent pas les $5 \%$, mais leur tendance est nettement visible : ils vont s'amplifier avec l'augmentation de l'épaisseur du film.

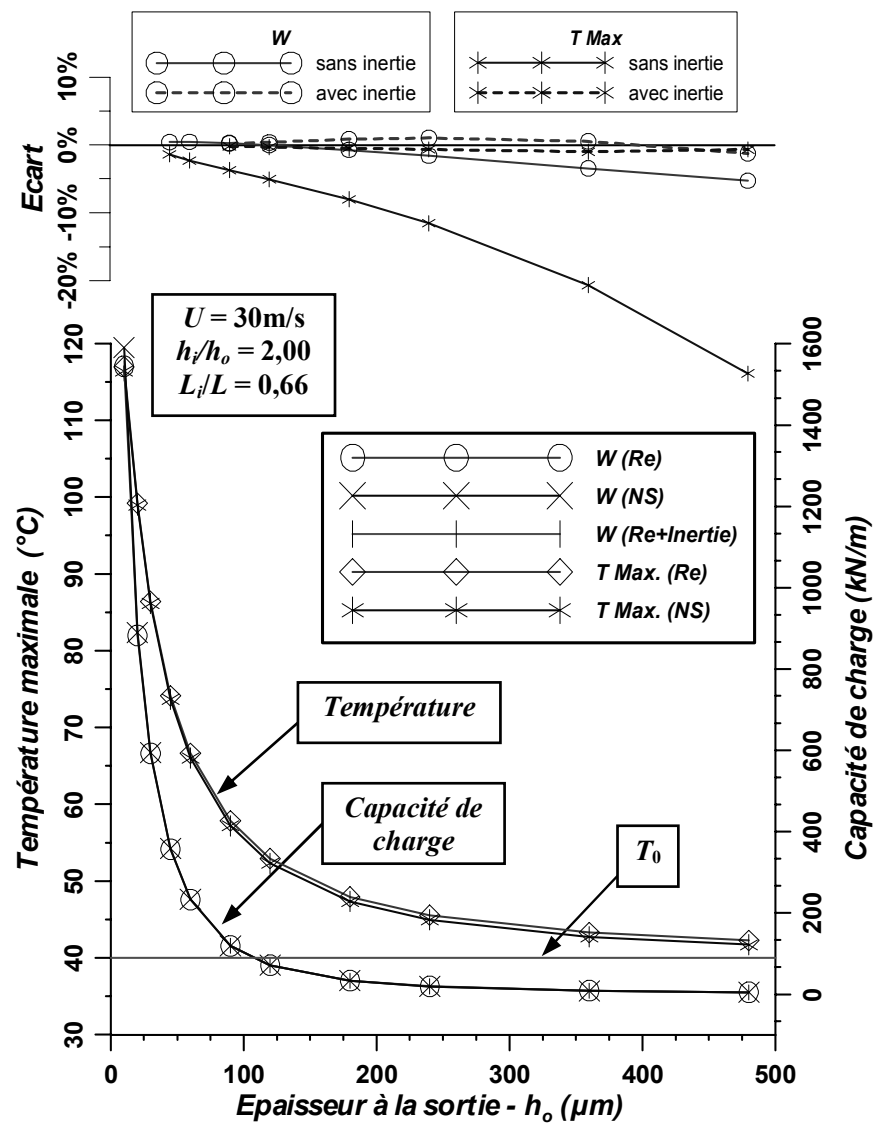

Figure 9. Influence de l'épaisseur du film (rapport $h_{\mathrm{i}} / h_{\mathrm{o}}$ maintenu constant)

L'introduction d'un modèle permettant l'approximation des effets d'inertie [4] lors de la résolution de l'équation de Reynolds généralisée (Re+Inertie) permet d'annuler presque la totalité de ces erreurs (représentées par des traits interrompus sur le graphique des écarts). Le coefficient de perte de charge utilisé dans cette étude est $\xi=0$. Une comparaison des champs de pression obtenus avec le modèle de Reynolds (avec et sans effets d'inertie) et le modèle de Navier-Stokes est présentée sur la figure 10. On note la perte de pression sur la ligne de discontinuité, prédite lorsque les effets d'inertie sont pris en compte (NS, Re+Inertie), mais totalement ignorée par le modèle de Reynolds classique $(\mathbf{R e})$. 


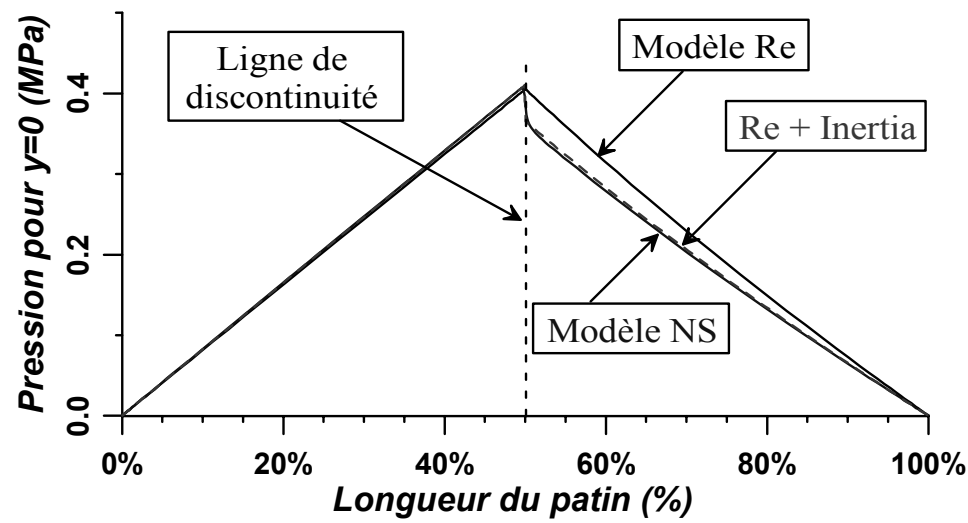

Figure 10. Pression sur la paroi mobile $\left(L_{i} / L=2, h_{i} / h_{o}=2, h_{o}=240 \mu \mathrm{m}, U=30 \mathrm{~m} / \mathrm{s}\right)$
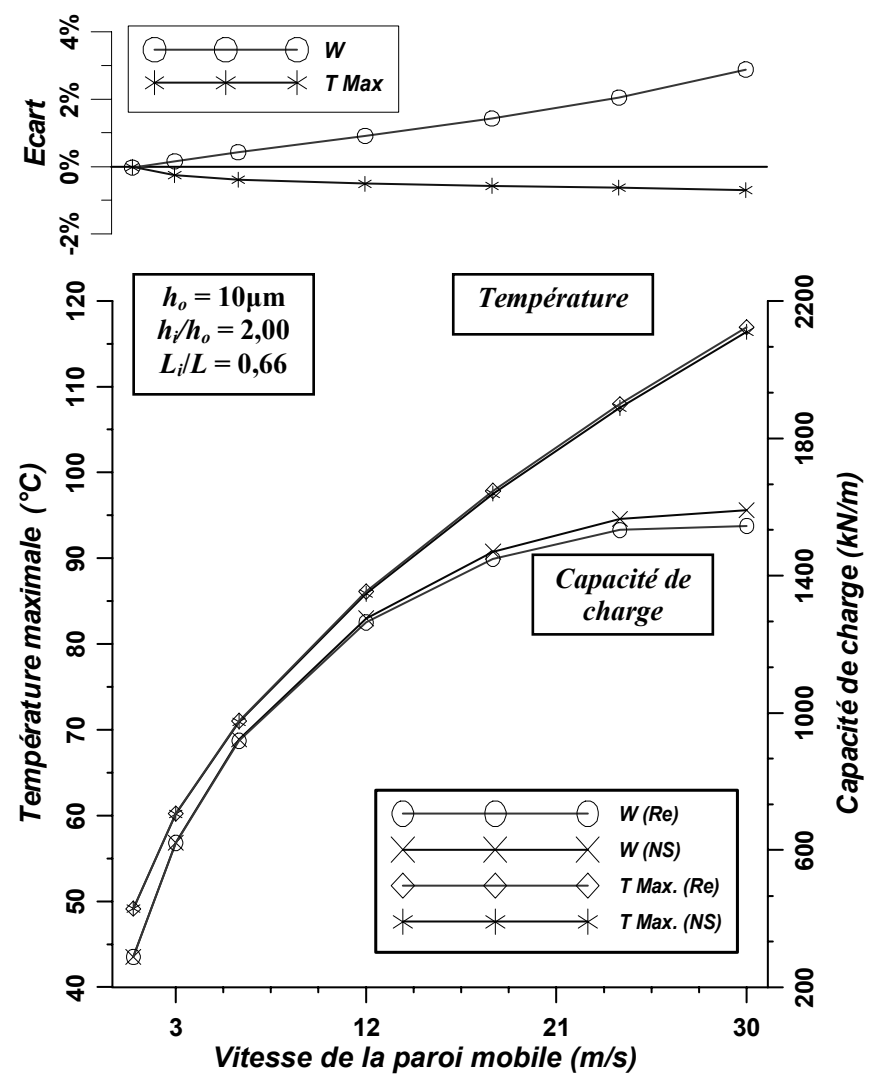

Figure 11. Influence de la vitesse de la paroi mobile $U$ 
Dans une dernière comparaison, la vitesse de la paroi mobile varie de 1 à $30 \mathrm{~m} / \mathrm{s}$ ( $R e=0,3 \div 28,0$ ), pour un film ayant une hauteur à la sortie $h_{\mathrm{o}}$ de $10 \mu \mathrm{m}$ et un rapport des longueurs $L_{\mathrm{i}} / L$ égal à 0,66 (figure 11). Les écarts sur la capacité de charge augmentent avec la vitesse, atteignant un maximum de $3 \%$ à $30 \mathrm{~m} / \mathrm{s}$. Cette fois, la prise en compte des effets d'inertie n'améliore plus la solution obtenue avec l'équation de Reynolds. En ce qui concerne la température maximale, les écarts entre les deux modèles restent faibles, autour de $1 \%$.

\section{Conclusion}

Dans cette étude, deux modèles numériques en volumes finis permettant l'analyse THD des films minces, discontinus, ont été proposés. Leur résolution fait appel aux techniques moins répandues dans la lubrification, telles que l'algorithme SIMPLE ou la méthode itérative de résolution des systèmes linéaires SIP. La solution basée sur les équations de Navier-Stokes a été considérée exacte et les écarts entre cette solution et celle obtenue avec le modèle de Reynolds ont été calculés pour plusieurs configurations d'un patin échelon. Les résultats suggèrent une bonne précision du modèle de Reynolds dans la plupart des cas. La prise en compte des effets d'inertie concentrée apporte plus de précision à ce modèle, permettant même l'étude des films épais. Les résultats obtenus ouvrent la voie aux analyses des systèmes présentant des discontinuités plus complexes, tels que les butées à poche et les paliers à saut, tout en conservant les hypothèses simplificatrices de l'écoulement des films minces.

\section{Nomenclature}

$\begin{array}{ll}C_{\mathrm{p}} & \text { Chaleur spécifique du fluide }\left[\mathrm{J} \cdot \mathrm{kg}^{-1} \cdot \mathrm{K}^{-1}\right] \\ E & \text { Epaisseur du patin [m] } \\ G, I_{2}, J_{2} & \text { Intégrales à travers l'épaisseur du film } \\ I_{1}, J_{1} & \text { Intégrales selon l'épaisseur du film } \\ h & \text { Epaisseur du film fluide }[\mu \mathrm{m}] \\ h_{\mathrm{a}} & \text { Coefficient de convection vers l'air }\left[\mathrm{W} \cdot \mathrm{m}^{-2} \cdot \mathrm{K}^{-1}\right] \\ h_{\mathrm{i}}, h_{\mathrm{o}} & \text { Epaisseur à l'entrée et à la sortie }[\mu \mathrm{m}] \\ K_{\mathrm{f}} & \text { Coefficient de conduction (fluide) }\left[\mathrm{W} \cdot \mathrm{m}^{-1} \cdot \mathrm{K}^{-1}\right] \\ K_{\mathrm{S}} & \text { Coefficient de conduction (patin) }\left[\mathrm{W} \cdot \mathrm{m}^{-2} \cdot \mathrm{K}^{-1}\right] \\ L & \text { Longueur du patin [m] } \\ L_{\mathrm{i}} & \text { Longueur du plan à l'entrée }[\mathrm{m}] \\ p & \text { Pression [Pa] } \\ p^{-}, p^{+} & \text {Pression avant et après la ligne de discontinuité }[\mathrm{Pa}] \\ T & \left.\text { Température [ }{ }^{\circ} \mathrm{C}\right]\end{array}$




$\begin{array}{ll}T_{\mathrm{a}} & \text { Température de l'air }\left[{ }^{\circ} \mathrm{C}\right] \\ T_{0} & \text { Température à l'entrée }\left[{ }^{\circ} \mathrm{C}\right] \\ T_{\text {moy }} & \text { Température moyenne dans le film }\left[{ }^{\circ} \mathrm{C}\right] \\ u, v & \text { Composantes de vitesse }\left[\mathrm{m} \cdot \mathrm{s}^{-1}\right] \\ u^{-}, u^{+} & \text {Vitesse moyennée avant et après la ligne de discontinuité }\left[\mathrm{m} \cdot \mathrm{s}^{-1}\right] \\ U & \text { Vitesse de la paroi mobile }\left[\mathrm{m} \cdot \mathrm{s}^{-1}\right] \\ x, y & \text { Coordonnées }[\mathrm{m}],[\mu \mathrm{m}] \\ W & \text { Capacité de charge }[\mathrm{N}] \\ \beta & \text { Coefficient de thermo-viscosité }\left[\mathrm{K}^{-1}\right] \\ \mu & \text { Viscosité du fluide }[\mathrm{Pa} \cdot \mathrm{s}] \\ \mu_{0} & \text { Viscosité à la température } T_{0}[\mathrm{~Pa} . \mathrm{s}] \\ \rho & \text { Densité du fluide }\left[\mathrm{kg} \cdot \mathrm{m}^{-3}\right] \\ \xi & \text { Coefficient de perte de charge }[-]\end{array}$

\section{Bibliographie}

Arghir M., Alsayed A., Nicolas D., "The Finite Volume Solution of the Reynolds Equation of Lubrication with Film Discontinuities", Int. J. of Mech. Sc., vol. 44, 2002, p. 2119-2132.

Boncompain R., Fillon M., Frêne J., "Analysis of Thermal Effects in Hydrodynamic Bearings", ASME J. of Tribology, vol. 108, 1986, p. 219-224.

Braun M.J., Choy F.K., Zhou Y.M., "The Effects of a Hydrostatic Pocket Aspect Ratio, Supply Orifice Position, and Attack Angle on Steady-State Flow Patterns, Pressure, and Shear Characteristics", ASME J. of Tribology, vol. 115, 1993, p. 678-685.

Braun M.J., Zhou Y.M., Choy F.K., "Transient Flow Patterns and Pressure Characteristics in a Hydrostatic Pocket", ASME J. of Tribology, vol. 116, 1994, p. 139-146.

Dowson D., “A Generalized Reynolds Equation for Fluid Film Lubrication”, Int. J. Mech. Sc., vol. 4, 1962, p. 159-170.

Ezzat H.A., Rhode S.M., "A Study of Thermohydrodynamic Performance of Finite Slider Bearings", ASME J. of Lubrication Tech., vol. 95, 1973, p. 298-307.

Fillon M., Khonsari M.M., "Thermohydrodynamic Design Charts for Tilting-Pad Journal Bearings", ASME J. of Tribology, vol. 118, 1996, p. 232-238.

Gandjalikhan N., Moayeri M.S., "Three-dimensional Thermohydrodynamic Analysis of Axially Grooved Journal Bearings”, I. Mech. E. (Part J), vol. 216, 2002, p. 35-47.

Han T., Paranjpe R.S., "A Finite Volume Analysis of Thermohydrodynamic Performance of Journal Bearings", ASME J. of Tribology, vol. 112, 1990, p. 557-566.

Hatakenaka K., Tanaka M., "Thermohydrodynamic Performance of Journal Bearings with Partial Reverse Flow and Finger-Type Cavitation", I. Mech. E. (Part J), 2002, vol. 216, p. 315-325. 
He M., Allaire P., Cloud C.H., Nicholas, J., "A Pressure Dam Bearing Analysis with Adiabatic Thermal Effects”, Tribology Trans., vol. 47, 2004, p. 70-76.

Keogh P.S., Gomiciaga R., Khonsari M.M., "CFD Based Design Techniques for Thermal Prediction in a Generic Two-axial Groove Hydrodynamic Journal Bearing”, ASME J. of Tribology, vol. 119, 1997, p. 428-436.

Patankar S.V., Numerical Heat Transfer and Fluid Flow, New York, Hemisphere, 1980.

Stone H.L., "Iterative Solutions of Implicit Approximations of Multidimensional Partial Differential Equations", SIAM J. Numerical Analysis, vol. 5, 1968, p. 530-558. 
